\title{
The transmission characteristics of the laser pulse in cumulus
}

\author{
Hongxia Wang ${ }^{1, a *}$ Qinghua Zhang ${ }^{1, b}$ Aijun $\mathrm{Li}^{1, \mathrm{c}}$ \\ ${ }^{1}$ Xi'an Research Inst. of Hi-Tech, Hongqing Town, Xi'an 710025, P.R. China \\ aredlightw@163.com, b15353652303@163.com, 'liaijun1979@163.com
}

Keywords: laser pulse; Multiple scattering; Time domain broadening; Monte Carlo; Cumulus

\begin{abstract}
The transmission of laser beams in dense medium like cumulus will produce multiple scattering phenomena. The results of multiple scattering not only weaken the laser energy, but also lead to the broadening of laser pulse in time domain. In this paper, the scattering parameters of cumulus were calculated based on the Mie scattering theory and the Gamma size distribution model. The Monte Carlo simulation model was established, and the corresponding characteristics of the energy attenuation and the time domain broadening have been analyzed, for the transmission of the laser pulse with four different wavelengths in cumulus. The relationship of the transmittance with the thickness of cumulus and the time distribution curve of the transmitted light were obtained. The research results demonstrate significant application in laser communication and laser radar.
\end{abstract}

\section{Introduction}

At present, the theory and application of laser in the field of national defense is researched intensify in the world. Laser communication, laser radar, laser guidance, or in the application of laser weapons, are involved in the transmission of laser in the atmosphere. Cloud and fog is a common natural phenomenon in the atmosphere, which has an important influence on the transmission of laser in the atmosphere ${ }^{[1]}$. The type and structure of cloud is very complex, for each kind of cloud, the height, thickness and particle size, attenuation coefficient, water content and so on are not the same. The particle radius and the optical depth of cloud are large, when laser passes through the clouds, it is prone to produce multiple scattering phenomena, which not only causes the laser energy attenuation, but also leads to the broadening of laser pulse in time domain. Therefore, the study on the transmission characteristics of laser in dense medium has been paid more and more attention. Based on the traditional single scattering method, the calculation error of the transmission characteristics of the laser in dense medium is large. In this paper, based on Monte Carlo method ${ }^{[2-4]}$, consider multiple scattering, the characteristics of energy attenuation and time domain broadening are computed and analyzed for four kinds of $\operatorname{laser}(0.53 \mu \mathrm{m}, 1.06 \mu \mathrm{m}, 3.8 \mu \mathrm{m}$ and $10.6 \mu \mathrm{m})$ transport in a typical cumulus.

\section{Extinction parameters of group particles}

When laser passes through the cumulus, scattering and absorption caused by cloud droplet particles are related with particle size distribution. There are many types of cumulus, its particle size distribution is quite complex, and has changed a lot with geography. According to experimental observation data, for a typical cumulus, the particle size distribution follows Gamma model as shown in the Eq.1:

$$
n(r)=2.604 r^{3} \exp (-0.5 r)
$$

Where, $r$ is particle radius, $n(r)$ is the number of particles in per unit volume and per unit radius interval, its unit is $\mathrm{cm}^{-3} \mu \mathrm{m}^{-1}$, the cloud droplet radius distribute between $0.1 \mu \mathrm{m} \sim 30 \mu \mathrm{m}$.

For the cumulus group particles, the total attenuation and absorption coefficient calculation formula are

$$
\mu_{i}=\int_{0}^{\infty} \sigma_{i}(r) n(r) d r \quad(i=t, a)
$$


Where, $\sigma_{t}$ and $\sigma_{a}$ are extinction and absorption cross section for a single particle respectively, its value can be calculated according to the Mie scattering theory:

$$
\begin{aligned}
& \sigma_{t}=\left(\lambda^{2} / 2 \pi\right) \sum_{n=1}^{\infty}(2 n+1)\left[\operatorname{Re}\left(a_{n}+b_{n}\right)\right] \\
& \sigma_{s}=\left(\lambda^{2} / 2 \pi\right) \sum_{n=1}^{\infty}(2 n+1)\left(\left|a_{n}\right|^{2}+\left|b_{n}\right|^{2}\right) \\
& \sigma_{a}=\sigma_{t}-\sigma_{s}
\end{aligned}
$$

Where, $\lambda$ is a wavelength, $a_{n}$ and $b_{n}$ are Mie scattering coefficient.

The calculation formula of average asymmetry factor is

$$
<g>=\int_{0}^{\infty} n(r) \sigma_{t}(r) g(r) d r / \int_{0}^{\infty} n(r) \sigma_{t}(r) d r
$$

According to the above equation and Mie scattering theory, the extinction parameter values of cumulus for laser are calculated as shown in table 1.

Table1. The extinction parameters of cumulus $\left(\mathrm{m}^{-1}\right)$

\begin{tabular}{lllll}
\hline Laser & $0.532 \mu \mathrm{m}$ & $1.06 \mu \mathrm{m}$ & $1.38 \mu \mathrm{m}$ & $10.6 \mu \mathrm{m}$ \\
\hline$\mu_{t}$ & 0.1304 & 0.1335 & 0.1351 & 0.1316 \\
$\mu_{a}$ & $6.6972 \times 10^{-8}$ & $5.2164 \times 10^{-5}$ & 0.0018 & 0.0527 \\
$g$ & 0.8664 & 0.8549 & 0.8530 & 0.9307 \\
\hline
\end{tabular}

\section{Monte Carlo calculation model for multiple scattering}

Monte Carlo method is based on probability model, through computer generated random numbers to simulate the random walk of a single photon and scattering in the medium, and think that photons interact with particles as the elastic scattering. Using Monte Carlo method, as long as the number of photons is enough, it can be used to simulate the multiple scattering of light.

Assumes that the laser along the $\mathrm{z}$ direction into the medium of the thickness of $L$ perpendicularly, photons movement step length is determined by the cumulative probability distribution of the mean free path, that is

$$
l=-\frac{1}{\mu_{t}} \ln \xi
$$

Where $\xi$ is a random number between the $(0,1)$.

After the photon and particle collision, the spatial distribution of the scattering angle is determined by Henyey-Greenstein phase function ${ }^{[5]}$, the sampling value of scattering angle $\theta$ is

$$
\theta=\cos ^{-1}\left\{\frac{1}{2 g}\left[(1+g)^{2}-\left(\frac{1-g^{2}}{1-g+2 g \xi}\right)^{2}\right]\right\}
$$

Where $g$ is the asymmetry factor $(0 \leq g \leq 1)$, reflects the anisotropic degree of scattering.

According to the weighted statistical estimation method, the photon is likely to be transmitted out at a certain probability in each collision point. Taking the $n$th photon for example, the probability of direct transmission out at the $i$ collision points is $P_{n}^{i}$, then

$$
P_{n}^{i}=\left\{\begin{array}{cc}
w_{i} \exp \left(-\mu_{t} \frac{L-x_{i}}{\cos \theta}\right) & \cos \theta>0 \\
0 & \cos \theta \leq 0
\end{array}\right.
$$


Where, $w_{i}$ is photon's weight at the $i$ th collision point: $w_{i}=w_{i-1} \exp \left(-\mu_{a} l_{i}\right)$. Initial photon weights $w_{0}=1$. When the photon's weight $w$ is smaller than a certain threshold (take $10^{-8}$ ), or the photon moves outside the media, that the photon die instead of tracking. After the termination of the photon, the simulation of the next photon is carried out until the simulation of all the photons is finished.

The direct sum of the probability in each collision point, that is, the contribution of the photon to transmittance

$$
P_{n}=\sum_{i=0}^{M-1} P_{n}^{i}
$$

Where, $M$ is collisions number of the $n$th photon. If a total of $\mathrm{N}$ photons are simulated, the estimated value of transmittance is all of the photon's statistical average contribution, then

$$
T=\frac{1}{N} \sum_{n=1}^{N} P_{n}=\frac{1}{N} \sum_{n=1}^{N} \sum_{i=0}^{M-1} P_{n}^{i}
$$

The transmission time of the photons in the cumulus is divided into many small intervals. The time broadening characteristics of laser pulse can be obtained by means of the statistics of photons in each cell.

\section{The calculation results and analysis}

According to the parameters in table 1, laser transmittances are calculated with the change of transmission distance in cumulus based on the Monte Carlo method. In the simulation, each tracking $\mathrm{N}=10^{6}$ photon, the result is shown in figure 1. Obviously, the transmittances of the laser of $3.8 \mu \mathrm{m}$ and $10.6 \mu \mathrm{m}$ wavelength sharp decrease with the increase of transmission distance in cumulus, the transmittances of $0.53 \mu \mathrm{m}$ and $1.06 \mu \mathrm{m}$ laser is significantly greater than the transmittance of $3.8 \mu \mathrm{m}$ and $10.6 \mu \mathrm{m}$ laser, the attenuation of cumulus is the weakest for $0.53 \mu \mathrm{m}$ laser, and the strongest for $10.6 \mu \mathrm{m}$ laser.

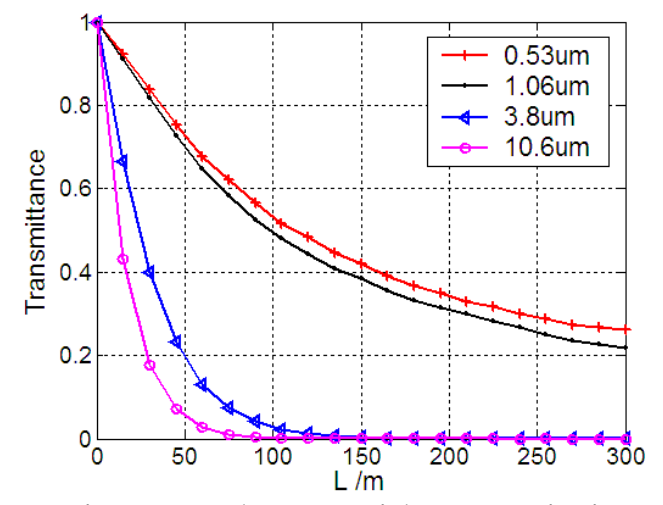

Fig.1 The transmittances change with transmission distance in cumulus

Laser transmission in the cumulus due to multiple scattering phenomena, each photon passes through the multipath propagation, resulting in the time of photon arrived at the output plane are not the same, which will lead to the phenomenon of time broadening of laser pulse. Figure 2 shows the time distribution of an ideal laser pulse in cumulus of three different thickness ( $\mathrm{L}=60 \mathrm{~m}, 90 \mathrm{~m}, 120 \mathrm{~m})$ based on Monte Carlo method. The results show that with the increase of the thickness of cumulus, laser pulse broadening is more obvious. When cumulus thickness is the same, the time domain broadening of $1.06 \mu \mathrm{m}$ laser is most significant, followed by $0.53 \mu \mathrm{m}$ and $3.8 \mu \mathrm{m}$ laser. The time domain broadening of $10.6 \mu \mathrm{m}$ laser is the weakest. Mainly because of the absorption coefficients of cumulus for $1.06 \mu \mathrm{m}, 0.53 \mu \mathrm{m}$ and $3.8 \mu \mathrm{m}$ laser are very small, scattering coefficient is relatively large, 
the multiple scattering phenomenon is more obvious, and absorption coefficient for $10.6 \mu \mathrm{m}$ laser is larger, the multiple scattering phenomenon is not significant.

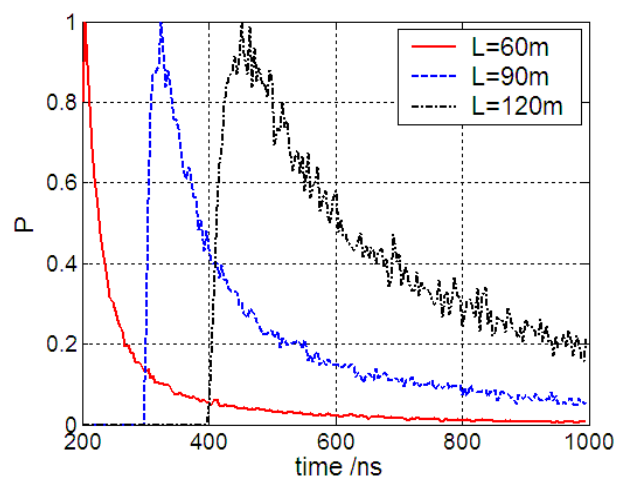

(a) $0.53 \mu \mathrm{m}$

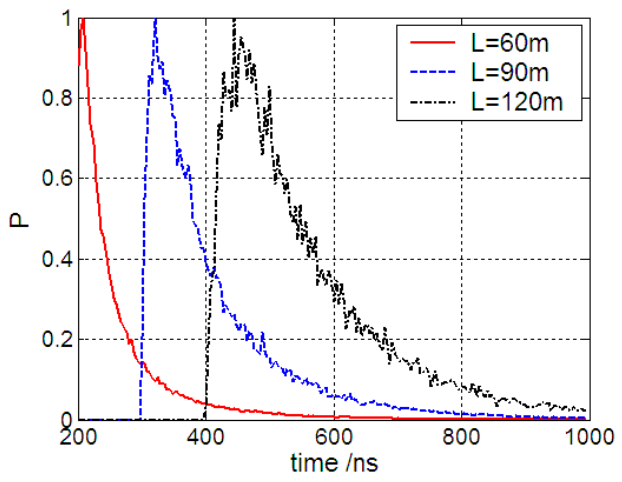

(c) $3.8 \mu \mathrm{m}$

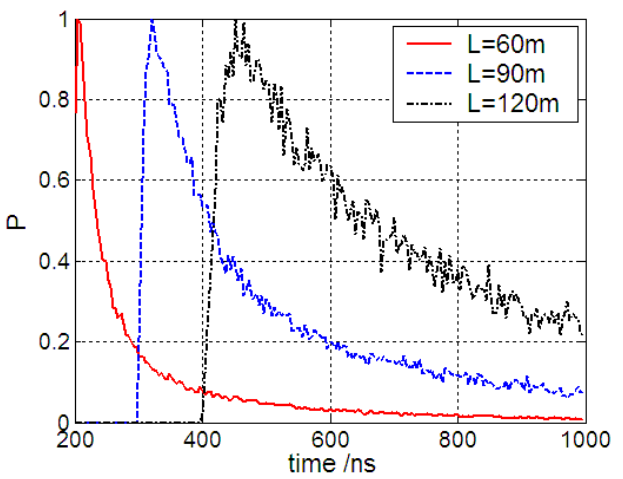

(b) $1.06 \mu \mathrm{m}$

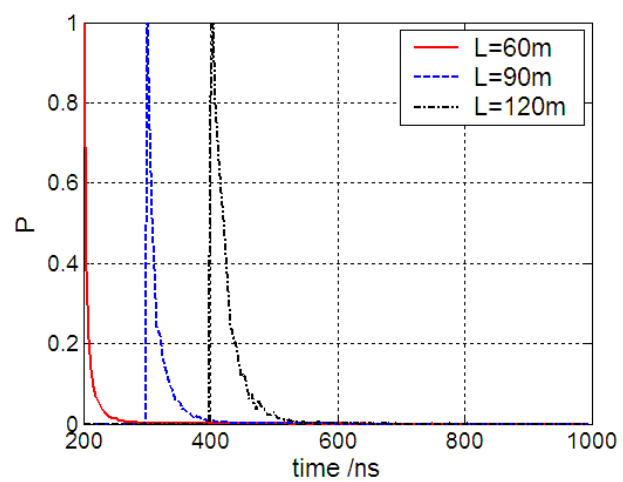

(d) $10.6 \mu \mathrm{m}$

Fig.2 The time distribution of transmitted laser with different wavelength

\section{Summary}

When laser pulse passes through the cumulus, the transmittances and time domain broadening are closely related to wavelengths of laser and thickness of cumulus. The attenuation of cumulus for $0.53 \mu \mathrm{m}$ and $1.06 \mu \mathrm{m}$ laser is weak, and is very strong for $3.8 \mu \mathrm{m}$ and $10.6 \mu \mathrm{m}$ laser. The time domain broadening of $10.6 \mu \mathrm{m}$ laser pulse transport in cumulus is very weak, and is very strong for the laser pulse with the wavelength of $0.53 \mu \mathrm{m}, 1.06 \mu \mathrm{m}$ and $3.8 \mu \mathrm{m}$.

\section{References}

[1] Adrian C. Selden. Attenuation and impulse response for multiple scattering of light in atmospheric clouds and aerosols, Appl. Opt. 13 (2006) 3144-3151

[2] D. E. Bates, J. N. Porte. AO3D: A Monte Carlo code for modeling of environmental light propagation, J. Quant.Spectrosc. Radiat.Transfer. 109 (2008) 1802-1824

[3] Balbas, E. M, P. J. French. Shape based Monte Carlo code for light transport in complex heterogeneous tissues, Opt. Express. 15 (2007) 14086-14098

[4] Bai, L., Z. S. Wu, S. Q. Tang, M. Li, P. H. Xie, and S. M. Wang. Study on phase function in Monte Carlo transmission characteristics of poly-disperse aerosol, Optical Engineering, 016002 (2011) 1-8

[5] Binzoni T,Leung T S,Gandjbakhche A H,et al.The use of the Henyey-Greenstein phase function in Monte Carlo simulations in biomedical optics, Phys .Med. Biol. 17 (2006) 313-322 